University of Wollongong

Research Online

Faculty of Science - Papers (Archive)

Faculty of Science, Medicine and Health

$1-7-2006$

\title{
The Role of the HPA Axis in Psychiatric Disorders and CRF Antagonists as Potential Treatments
}

Paul A. Keller

University of Wollongong, keller@uow.edu.au

\author{
A. McCluskey \\ University of Newcastle \\ J. Morgan \\ University of Wollongong, jodym@uow.edu.au
}

S. M. O'Connor

University of Wollongong

Follow this and additional works at: https://ro.uow.edu.au/scipapers

Part of the Life Sciences Commons, Physical Sciences and Mathematics Commons, and the Social and Behavioral Sciences Commons

\section{Recommended Citation}

Keller, Paul A.; McCluskey, A.; Morgan, J.; and O'Connor, S. M.: The Role of the HPA Axis in Psychiatric Disorders and CRF Antagonists as Potential Treatments 2006.

https://ro.uow.edu.au/scipapers/30

Research Online is the open access institutional repository for the University of Wollongong. For further information contact the UOW Library: research-pubs@uow.edu.au 


\title{
The Role of the HPA Axis in Psychiatric Disorders and CRF Antagonists as Potential Treatments
}

\author{
Abstract \\ An overview of the links between the Hypothalamic-Pituitary-Adrenal (HPA) axis and psychiatric disorders \\ is presented. The current treatments are outlined, indicating that they are insufficient to meet the needs of \\ those that suffer from these affective disorders. Therefore, there is an urgent need for the generation of \\ new therapeutics, in particular, against new targets. The association of the corticotrophin releasing factor \\ (CRF) and the HPA axis indicates that CRF antagonists should be beneficial as potential therapeutics.

\section{Keywords} \\ Medicinal Chemistry, Psychiatric disorders, corticotrophin releasing factor, therapeutics, CMMB

\section{Disciplines} \\ Life Sciences | Physical Sciences and Mathematics | Social and Behavioral Sciences

\section{Publication Details} \\ This article was originally published as: Keller, PA, McCluskey, A, Morgan, J \& O'Connor, SMJ, The Role of \\ the HPA Axis in Psychiatric Disorders and CRF Antagonists as Potential Treatments, Archiv der \\ Pharmazie, 2006, 339(7), 346-355. Copyright 2006 John Wiley \& Sons. Journal information available here.
}


The Role of the HPA Axis in Psychiatric Disorders and CRF Antagonists as Potential Treatments

\section{Review}

Paul A. Keller, ${ }^{a}$ Adam McCluskey, ${ }^{\mathrm{b}}$ Jody Morgan, ${ }^{a}$ Sean M. J. O’Connor ${ }^{\mathrm{a}}$

${ }^{a}$ Department of Chemistry, University of Wollongong, Wollongong 2522, Australia

${ }^{\mathrm{b}}$ Chemistry, School of Environmental and Life Sciences, University of Newcastle, Callaghan, 2308, Australia.

Running title: CRF Antagonists as Treatment for Psychiatric Disorders

Corresponding Author:

Paul A. Keller

Department of Chemistry

University of Wollongong

Wollongong 2522

Australia

Telephone: +61242214692

Fax: + 61242214287

Email: keller@uow.edu.au

Key Words: Psychiatric disorders, corticotrophin releasing factor, therapeutics 


\section{Summary}

An overview of the links between the Hypothalamic-Pituitary-Adrenal (HPA) axis and psychiatric disorders is presented. The current treatments are outlined, indicating that they are insufficient to meet the needs of those that suffer from these affective disorders. Therefore, there is an urgent need for the generation of new therapeutics, in particular, against new targets. The association of the corticotrophin releasing factor (CRF) and the HPA axis indicates that CRF antagonists should be beneficial as potential therapeutics.

\section{Introduction}

It is estimated that $22 \%$ of Americans aged 18 years and older suffer from some form of diagnosable mental disability [1]. Of the 10 leading causes of disability in the US, four are psychiatric diseases and include unipolar disorder (UPD), bipolar disorder (BPD), anxiety and anorexia nervosa [2]. These figures have been mirrored in other developed countries such as the United Kingdom and Australia where approximately $20 \%$ of the adult population suffer from some form of psychiatric disability [3].

Current treatments for these conditions are barely adequate and there is the need for the development of a new generation of novel psychiatric pharmaceutical agents acting via alternate mechanisms. Several new hypotheses have been formulated in recent times in response to the ongoing inadequacy of current psychiatric treatments. Many of these directly suspect the dysregulation of the bodies stress system, the hypothalamic-pituitary-adrenal (HPA) axis, as being responsible for the induction and prolongation of psychiatric diseases [4]. Subsequently these hypotheses have implicated the HPA axis components; corticotropin-releasing factor (CRF), glucocorticoids and cyclic adenosine monophosphate (cAMP) response element binding proteins (CREB) as subsystems that are potentially responsible for psychiatric malfunctions [5].

Although the HPA axis has been studied extensively since the 1950's, only recent breakthroughs in biotechnology and endocrinology have resulted in the successful cloning of CRF and its receptors [6]. Thus allowing the means to closely study and monitor their characteristics.

Recent studies [7] analysing the HPA axis and its involvement in psychiatric diseases have identified the following unusual symptoms in patients; high concentrations of CRF in the central nervous system (CNS), abnormal results to corticoid response tests, hyperactive CRF neuron activity and abnormal CRF receptor expression patterns. This has justified initial scientific efforts to 
look more closely at the role of CRF in the induction HPA dysregulation [7]. The increasing evidence that CRF and the HPA axis play definitive roles in many psychological disabilities has led to a focus on CRF antagonists as a novel means to treat these disorders [8].

This review will discuss the current knowledge of CRF and its receptors, examine evidence put forward to support the CRF-HPA dysregulation hypothesis while finally commenting on recent developments in CRF antagonist research.

\section{Psychiatric Disabilities}

Undoubtedly an extensive range of psychiatric conditions will potentially benefit from CRF based antagonist treatment. Even though a wide range of psychiatric disorders exist, discussion will focus on affective, anxiety and narcotic dependence based conditions.

\section{Affective disorders:}

Affective disorders are the most common form of psychiatric condition present in modern day society [9]. While often being a preluding or partnered condition for numerous other disorders such as drug addiction, obsessive compulsive disorder (OCD), anxiety and anorexia, affective disorders are generally classified as depression, which is further divided into two subtypes; bi-polar and unipolar disorders.

Bi-polar disorder, more commonly known as manic depression, is a form of mood disorder characterised by brief periods of euphoria followed by alternating periods of severe depression. The manic or euphoric phases are characterised by extreme hyperactivity, restlessness and feelings of great self importance often accompanied by a denial of their condition $[7,9,10]$. The disease occurs in approximately $1.6 \%$ of the world population and is diagnosed equally between men and women $[10]$.

Uni-polar depression differs from its bi-polar counterpart as no mania spells are experienced by the patient. Depressive episodes follow a more continuous unrelenting pattern with intermittent periods of normal emotional behaviour. Additional symptoms include torpidity due to diminished energy levels, low self esteem and reduced motivation to partake in normal activities [7,9]. This can lead to a breakdown of social and family ties and reduced productivity in the workforce, with an estimated cost in the US alone of $\$ 55$ billion per year arising from depression-related illnesses [10]. 
Anxiety:

Anxiety disorders encompass a wide range of conditions such as general anxiety disorders (GAD), panic disorder, post traumatic stress disorder, phobias and Tourette syndrome [9, 11]. These disorders are characterized by patients experiencing fear or constant episodes of anxiety over matters which do not necessarily require or provoke any real cause for anxiety. Anxiety disorders cause symptoms such as chest pains, dizziness and hot flashes during panic attack episodes, increased heart rate, poor concentration and irrational behaviour. One in every eight Americans aged 18-54 suffers from an anxiety disorder [7], with these statistics being mirrored in other developed nations.

\section{Substance abuse:}

Drug addiction is defined as the uncontrollable desire to take a drug accompanied by diminished control in limiting its intake [12]. Drug addiction, also known as drug dependency, can involve both physiological and psychological dependence. Physiological dependence is caused by alterations to the user's physiology and metabolic pathways resulting in adaptation requiring the drug to be present for continued function. Psychological dependence is when patients assume they need a drug in order to properly function where in fact no physiological dependence exists [11, 12]. Drug addiction is usually more prevalent in males than females however the incidence of female addiction is rising [12].

Narcotic addiction or dependence can result in increased tolerance barriers by physiological adaptations by the brain, e.g. desensitisation through reduction in receptor populations [13]. Unfortunately this can lead to the use of high doses of narcotic substances which can give rise to permanent organ damage and/or death. Some of the known addiction or dependency side effects include; social isolation, poor concentration, aggressive behaviour and irrational thinking.

In the US, 23 million adults are believed to be repeat substance offenders [7, 10]. Drug addiction in the US is estimated to cost over $\$ 67$ billion dollars a year in social and medical costs for treatment of illicit drug users [12].

In summary affective, anxiety and substance abuse are three classes of psychiatric disorders with unique mechanisms. There is growing evidence to support that these three conditions share a common involvement of CRF and the HPA.

\section{Current Treatments of Psychiatric Disorders}


Current treatments have been designed according to the monoamine theory, whereby focus is placed on regulating the production and reuptake of neurotransmitters such serotonin, norepinephrine and dopamine, which have been attributed to play a significant role in depression [14]. Unfortunately, as well as being effective, these agents also have numerous limitations. Initial treatment only cures approximately $50 \%$ of patients with one in three not responding to standard treatments in the long term [15].

Selective Serotonin Reuptake Inhibitors (SSRIs) are currently the most prescribed drug family for treatment against depression and anxiety related illnesses [16]. Typically SSRI drug therapy takes 4-6 weeks to show any positive results, and is preferred for depression patients as the risks associated with overdosing are greatly reduced $[16,17]$. Once symptoms subside, antidepressant therapy is continued for up to 6-9 months [15-17]. Such lengthy medication regimes are expensive and patient discipline in continuing treatment for this long can waver [16]. SSRI regimes are unfortunately associated with draw backs such as nausea, insomnia, and low libido which can impede sexual functions [18].

Monoamine Oxidase Inhibitors (MAOIs) were the first antidepressants introduced into mainstream society and have also been adopted to treat anxiety patients. These drugs are still considered secondary option treatments for patients whom fail to respond to alternative first wave therapeutics for anxiety and depression [16]. MAOIs initiate the rapid and sustained release of serotonin (5-HT) in the brain by inhibiting monoamine oxidase. Common side effects associated with MOAI therapy are; hypotension, tremors, insomnia, convulsions while in some cases they can induce dangerous drug-to-drug and drug-food interactions causing serious harm $[18,19]$. As a result patients are often forced to comply with strict diets and are prevented from taking many other pharmaceutical agents [19].

Tricyclic antidepressants (TCA) drug therapy is known to have similar results to SSRI treatments [17], however they usually require a longer period for their onset of action compared to SSRI based drugs [15]. Their method of action is primarily to prevent the reuptake of amines by nerve terminals. TCA therapies typically cause blurred vision and constipation. In addition, patients with heart complications are strictly prohibited from using these drugs as they can cause arrhythmias $[20]$.

Beta-Blockers and benzodiazepines are fast-acting short term anxiety treatment options requiring unfavourable repeated dosing applications to prolong therapeutic effects [16]. Beta-Blockers are not 
prescribed to patients with asthma or heart complications as they can amplify these conditions [21]. Benzodiazepines are addictive and cause drowsiness, fatigue and poor memory making their usage highly unfavourable [22].

\section{Corticotropin Releasing Factor (CRF)}

CRF is the hypothalamic peptide Fig. (1) that stimulates adrenocorticotropin hormone (ACTH) release from the pituitary gland which in turn induces cortisol release as a mechanism for organisms to deal with stress inducing situations [23]. CRF is a small 41 amino acid peptide synthesized and released from multiple regions of the brain including the paraventricular nucleus (PVN), hypothalamus and the locus ceruleus (LC) [24]. CRF is the initiator of the HPA axis and determines the rate at which the HPA axis operates. This peptide has also been associated with secondary roles such as the regulation of proopiomelanocortin (POMC) and other neuronal peptides. [25]

\section{H-Ser-Glu-Glu-Pro-Pro-Ile-Ser-Leu-Asp-Leu-Thr-Phe-His-Leu-Leu-Arg-Glu-Val-Leu-Glu-Met- Thr-Lys-Ala-Asp-Gln-Leu-Ala-Gln-Gln-Ala-His-Ser-Asn-Arg-Lys-Leu-Leu-Asp-Ile-Ala- $\mathrm{NH}_{2}$}

Figure 1: Amino acid sequence of CRF.

\section{Distribution of CRF:}

CRF synthesis and storage bodies are densely populated within the paraventricular nucleus (PVN) of the hypothalamus and amygdala [26]. Production of CRF is stimulated through various neuronal peptides and transmitters including acetylcholine, histamine, serotonin and many other common neuronal messengers [27]. The PVN and amygdala neuronal bodies distribute their axons to the capillaries in the median eminence, lower brain steam, cerebral cortex and spinal cord [28]. High concentrations of CRF are present in the locus caeruleus and the central nucleus of the amygdala, both of which are involved in anxiety and stress behaviour regulation and are stimulated by CRF neurons in the PVN. [4, 23, 29]. Additional CRF containing bodies are present in the neocortex and bed nucleus of the stria terminalis [29].

Peripheral tissues including the stomach, pancreas, small intestine, lymphocytes, placenta and the testes also have demonstrated a significant CRF presence [23]. The identification of CRF in these non-CNS neuronal bodies suggests its wide range of influence in numerous physiological systems such as immunity, digestion and reproduction in addition to the stress axis [26-28]. 


\section{CRF Receptors:}

Currently 2 types of CRF receptors have been classed and identified, $C_{R} F_{1}$ receptors $\left(C R F_{1} R\right)$ and $\mathrm{CRF}_{2}$ receptors $\left(\mathrm{CRF}_{2} \mathrm{R}\right)$. Both receptors have their own unique gene and custom distribution within the body. $\mathrm{CRF}_{1} \mathrm{R}$ and $\mathrm{CRF}_{2} \mathrm{R}$ both share close homology with one another and belong to the class $\mathrm{B}$ subtype of G-protein coupled receptors Fig. (2) [28].

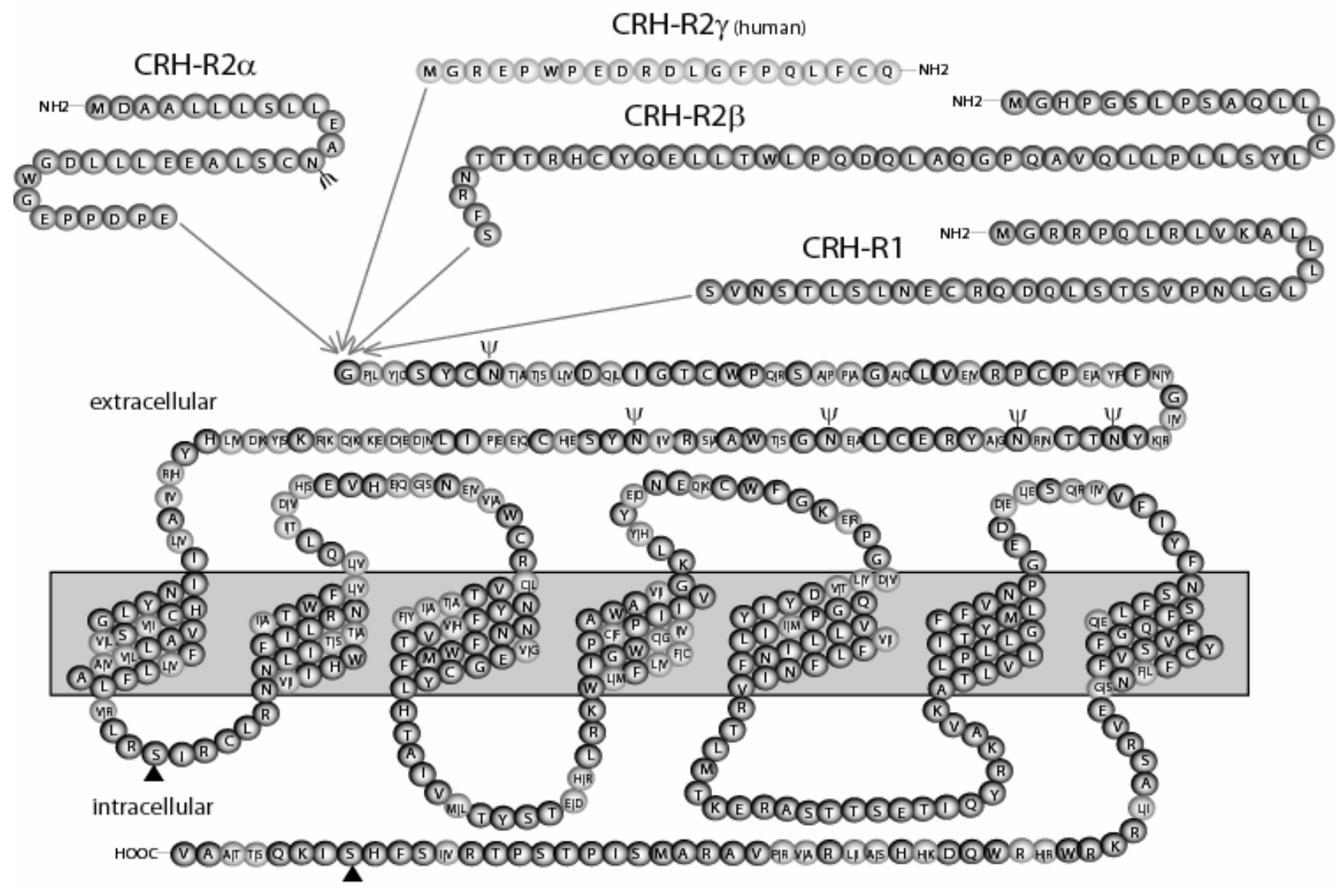

Figure 2: Amino acid sequences for corticotropin releasing hormone $\mathrm{CRF}_{1}, \mathrm{CRF}_{2 \alpha}, \mathrm{CRF}_{2 \beta}$ and $\mathrm{CRF}_{2 \gamma}$ receptor types. The seven transmembrane domains are shown where the arrows indicate the divergence between the $\mathrm{CRF}_{1}$ and the various $\mathrm{CRF}_{2}$ receptors. Glycolysation sites have been indicated by $\psi$ while $\boldsymbol{\Delta}$ indicate protein kinase $\mathrm{C}$ binding sides.

The third intracellular loop within all CRF receptors is thought to be the interactive region between the receptor and the coupled G-protein. This hypothesis is supported by the fact that the third intracellular loop is identical across all isoforms of the CRF receptor [26, 28]. CRF receptors are positively regulated via cAMP accumulation in response to CRF agonist binding which then relays the hormonal signal [29]. 
The $\mathrm{CRF}_{1} \mathrm{R}$ is 415 amino acids in length, its seven transmembrane domains contain $5 \mathrm{~N}$-linked glycolysation sites and 2 potential phosphorylation sites for protein kinase $\mathrm{C}$ (PKC) in the Cterminal tail. $\mathrm{CRF}_{1} \mathrm{R}$ also has casein kinase II and protein kinase Fig. (2). A phosphorylation sites in the $3^{\text {rd }}$ extracellular loop, however their purpose remains unclear [30]. A variety of $\mathrm{CRF}_{1} \mathrm{R}$ spliced variants exist and have been classified $\mathrm{CRF}_{1 \mathrm{a}}$ through to $\mathrm{CRF}_{1 \mathrm{~h}}$ [4]. These variants have not shown any significant alternative activity compared to $\mathrm{CRF}_{1 \mathrm{a}} \mathrm{R}[4]$.

$\mathrm{CRF}_{1} \mathrm{R}$ shows high distribution within the brain and other sections of the CNS. Most dense areas of distribution are in the cerebral cortex, olfactory bulb, medial septum, hippocampus, amygdala and the pituitary $[29,31,32]$. Through autoradiography localisation studies it has been discovered that $\mathrm{CRF}_{1} \mathrm{R}$ distribution patterns in different compartments of the brain vary [31]. For instance $\mathrm{CRF}_{1} \mathrm{R}$ within the anterior pituitary seem to be clustered, mirroring the distribution of corticotrophs. This contrasts $\mathrm{CRF}_{1} \mathrm{R}$ distribution within the intermediate lobe where the receptors are localised evenly across the lobe, following the distribution patterns of POMC producing cells. [33]

The distribution patterns of $\mathrm{CRF}_{1} \mathrm{R}$ within the brain and peripheral tissues support current hypotheses suggesting CRF is responsible for POMC peptide regulation and secretion from the anterior and intermediate pituitary lobes [23,33].

\section{$C R F_{2}$ Receptors $\left(C R F_{2} R\right)$ :}

The second splice variant of the CRF receptor family, $\mathrm{CRF}_{2} \mathrm{R}$, can be subdivided into 3 isoforms $\mathrm{CRF}_{2 \alpha} \mathrm{CRF}_{2 \beta}$ and $\mathrm{CRF}_{2 \gamma}$. Both $\alpha$ and $\beta$ isoforms have been isolated in rats, mice and humans however $\mathrm{CRF} 2{ }_{2 \gamma} \mathrm{R}$ has only been identified in humans $[26,34]$.

$\mathrm{CRF}_{2}$ receptors, unlike their $\mathrm{CRF}_{1} \mathrm{R}$ counterparts, have a wide distribution, encompassing a broader range of tissues. Studies showed $\mathrm{CRF}_{2} \mathrm{R}$ mRNA is expressed in the lateral septal nuclei, hypothalamic nuclei, in the bed nucleus of the stria terminals along with amygdaloid nuclei, though not at the same intensity as $\mathrm{CRF}_{1} \mathrm{R}$ [31]. Furthermore these studies went on to establish that $\mathrm{CRF}_{2 \gamma} \mathrm{R}$ are distributed mainly in areas of the $\mathrm{CNS}$ whereas the $\mathrm{CRF}_{2 \alpha} \mathrm{R}$ and $\mathrm{CRF}_{2 \beta} \mathrm{R}$ have been identified primarily in non-neuronal and peripheral tissues such as the cerebral arterioles and the choroid plexus of the ventricular system [34]. $\mathrm{CRF}_{2 \beta} \mathrm{R}$ are also found in the heart, GI tract, skeletal muscle and lungs $[4,23]$. Due to the dense distribution of $\mathrm{CRF}_{2 \beta} \mathrm{R}$ within the cerebral arterioles and the choroid plexus of the ventricular system it leads to the potential hypotheses that $\mathrm{CRF}_{2 \beta} \mathrm{R}$ are in fact linked to a modulation role for cerebral blood flow [33]. 


\section{CRF from gene to protein:}

The human CRF gene is assembled by 2 exons 686-800 base pairs in length and has been mapped on Chromosome 8 (8q13) [23]. The translation of the CRF gene produces a 196 amino acid (aa) pre-proCRF molecule which is enzymatically treated within the rough endoplasmic reticulum to form proCRF which undergoes additional post-translational alterations [35, 36]. Further alterations are performed in the trans-Golgi network to produce the final 41 aa CRF peptide [36].

CRF production is regulated through the protein kinase pathway. Studies where cAMP has been administered to perfused rat hypothalami have shown marked increases in CRF secretion [31]. cAMP is thought to interact with a cAMP responsive element (CRE) region approximately 200 base pairs upstream from the CRF gene, promoting gene translation $[35,36]$. Examination of CRF and the HPA axis has shown that CRF initiated release of glucocorticoids from the adrenal glands results in a negative feedback regulation mechanism, where glucocorticoids inhibit CRF production by interfering with cAMP-response element binding protein CREB/CRE controlled gene transcription process [35-37].

\section{HPA Axis and stress response:}

The HPA axis is the infrastructure of the body which regulates the stress response. CRF neurons within the PVN of the hypothalamus are the primary source of CRF within the CNS. Release of CRF stimulates the anterior pituitary to release $\mathrm{ACTH}$, which is transported through the blood stream to the adrenal cortex to initiate the synthesis and secretion of cortisol, a glucocorticoid [38].

Regulation of the HPA axis is controlled by an intricate network of neuronal and hormonal pathways including the hippocampus, the amygdala, glucocorticoid peptides and monoamine neurotransmitters $[38,39]$. Activation of the HPA axis results in higher locomotive activity, reduced sex drive, low affinity for food, redirected blood flow from the gastro intestinal tract to skeletal muscle, increased sensory sensitivity, increased heart rate and raised blood sugar levels. These adaptations which help an individual respond to a stress event $[39,40]$.

A stress event lasting longer than a few minutes results in increased levels of cortisol being released from the adrenal cortex. $\mathrm{CRF}$ and $\mathrm{ACTH}$ in healthy individuals are released only in short intermittent bursts resulting in controlled cortisol release [41]. Thus, in stressful situations, a moderate cortisol level is maintained in the bloodstream to main stable physiological function and homeostasis in the HPA axis [41]. 
A stress event may be emotionally, physically or chemically induced which initiates CRF secretion over the affected time period, allowing for beneficial physiological adaptations i.e. increased sensory sensitivity which allows individual organisms to respond adequately to a stressful situation [39]. Prolonged stress influences are thought to over-stimulate the HPA axis causing hypersecretion of CRF which ultimately, if left untreated, leads to HPA dysregulation potentially promoting the onset of psychiatric disorders [41].

\section{Support for HPA Dysregulation Theory}

Numerous studies have indicated that individuals suffering from anxiety and/or depression possess a sustained unregulated HPA axis. The hyperactivity of the HPA axis has been shown to exacerbate the additional secretion of CRF and subsequently ACTH. This dysregulation is the assumed cause of hypercortisolemia, a symptom observed in numerous psychiatric related disabilities [38].

\section{Post Mortem Examinations:}

Post mortem analysis of brain tissues obtained from suicide victims who were suffering from long term depression showed a significant reduction in CRF receptor sites in various regions of the brain, most noticeably in the cerebral cortex [4]. The emerging pattern of reduced receptor sites suggested the presence of a mechanism attempting to compensate for the hypersecretion of CRF [42]. Further study into this phenomenon led to the discovery that suicide victims also had a $400 \%$ increase in CRF producing neurons within the PVN and a dramatic increase in CRF mRNA expression compared to standard controls [43]. Therefore a mechanism in depressed patients exists which is responsible for the recruitment of additional neuronal cells to produce CRF. This suggests dysregulation in the HPA axis as CRF secretion is uninhibited in these patients [44]. Such drastic increases of CRF secretion would no doubt lead to higher concentrations of ACTH and cortisol in the blood stream and CNS.

\section{CRF in cerebral spinal fluid (CSF) and blood plasma:}

Following initial indications that CRF might be involved in depression and anxiety disorders [45] further analyses of CRF concentrations were employed. Due to the difficulty of obtaining CRF concentration data directly from the brain, cerebral spinal fluid (CSF) was used to gauge CRF concentrations where a clear correlation between diseased states and CRF concentrations in the CSF was established [46]. Results showed there was a tangible increase in CSF CRF concentrations in patients who were suffering from depression, anxiety and dementia when compared to healthy controls $[45,46]$. In addition results have also indicated increased levels of CRF in the blood stream among depressed patients [23], however these results have not been replicated in follow up studies 
[33, 45-46]. The increased amount of CRF discovered in varying bodily fluid systems across different psychiatric diseases suggests the presence of CRF hypersecretion, thus indicating a dysregulated HPA axis.

\section{Anxiety effects of CRF:}

CRF neurons are believed to encompass numerous additional functions including the influence on proopiomelanocortin (POMC) synthesis through the release of CRF into the portal blood stream [47]. POMC is converted to melanocortin which is known to inhibit feeding behaviour and induce weight loss [47-49]. When an excess of POMC is produced the side effects mimic those observed in anorexia nervosa patients [47-49]. Observation of rats undergoing chronic exposure to CRF indicated the manifestation of several response adaptations including hyperexcitability, fear and other anxiogenic behaviours normally associated with anxiety [50-52]. These initial trials were then further extended by injecting urocortin, a CRF receptor agonist, into central nucleus of the amygdala, hypothesised as being the key body involved in signal transmissions responsible for the onset of anxiety disorders [51]. Results provided evidence of severe anxiogenic symptoms as a direct result of the chronic agonist exposure.

Antisense oligodeoxynucleotide (ODN's) tests provided the opportunity to observe if non-CRF producing rats were still liable to suffer from stress or anxiety behaviour [53]. ODN exploited rats had significantly decreased anxiety like behaviour and showed a reduction in stress. In reply to these investigations an alternative approach was trialled whereby CRF was over-expressed using transgenic mice, mimicking the excess CRF secretion causing HPA dysregulation as observed in psychiatric patients. As expected, the over-production of CRF in the transgenic mice caused severe anxious behaviour [52-53]. Extended trials then observed the effect of CRF antagonists upon these test subjects. As hoped a reversal of anxiety and depression symptoms occurred [23, 33]. By creating a CRF hypersecretion model in rodents reflecting human observations, these experiments were able to provide strong supporting evidence linking anxiety to HPA-CRF dysregulation.

\section{Effects of current pharmaceutical agents on CRF:}

It has been indicated that current antidepressants may produce their therapeutic effects partly through the regulation of the HPA-CRF system [14]. Such a hypothesis was first considered when exposing rats to imipramine, a tricyclic antidepressant (TCA) and CP-154526, a $\mathrm{CRF}_{1} \mathrm{R}$ antagonist, resulted in decreased immobility indicating a reduction of CRF influence was linked to a reversal of anxiety and depression through classical treatments [20]. These trials were further expanded where it was discovered that rats which had undergone imipramine treatment for two weeks showed signs 
of increased glucocorticoid receptor (GR) immunoreactivity [17]. This change allowed for an increase in the glucocorticoid negative feedback mechanism in the HPA axis resulting in a reduction of CRF mRNA in various regions of the brain most importantly the hippocampus, hypothalamus and the pituitary [40].

Additional evidence proposing alternate mechanisms of action came to light when high numbers of CRF receptors were observed in rat brains which had undergone chronic imipramine exposure, suggesting that CRF production had been impeded, forcing the body to compensate by increasing binding sites $[15,17]$. Alternatively links between antidepressant use and a reduction in CRFR in the anterior pituitary were also established when reductions of $\mathrm{CRF}_{1} \mathrm{R}$ mRNA expression in the amygdala was observed in rats after exposure to SSRI treatment [14]. Further studies subsequently indicated the increase in GR mRNA expression in the hippocampus caused a suspected increase in HPA axis inhibition as a result of antidepressant usage [16].

Similar studies have been performed on current anti-anxiety treatments, implying they inhibit the HPA axis. Acute benzodiazepine administration has been shown to result in reduction in CRF concentration in the locus coeruleus, amygdala and the pyriform cortex, all of which are associated with stress behaviour [29].

Evidence provided in these monoamine studies has identified many inconsistencies in monoamine theory, on which most modern day antidepressants and anti-anxiety medications are founded upon $[14,16]$. Most notably, compounds known to significantly enhance monoamine transmission, which is the suspected mechanism of action of monoamine based treatments, had little to no therapeutic effect in depressed or anxiety patients. Neurotransmitter concentrations between groups taking standard medications and the new transmission enhancing drugs were similar, however the new compounds had no therapeutic effect $[14,16,17]$. This suggests that current antidepressant and anti-anxiety medications do not enact their therapeutic benefits through monoamine pathways. In addition it is yet to be explained why certain clinical treatments currently in use show minimal effects on monoamine transmission pathways, though are potent and therapeutically effective against depression and anxiety. Furthermore some antidepressant drugs have delayed therapeutic benefits which, interestingly coincides with an apparent inhibition rather than excitatory effects on monoaminergic transmission [16]. 
These conflicting results suggest that perhaps the current therapies are not primarily enacting their therapeutic properties through monoamine neuropeptide pathways but also via other mechanisms related to HPA regulation.

\section{Evidence of HPA dysregulation in substance abusers:}

Considerable evidence has indicated that acute administration of psychostimulants causes a stress like activation of the HPA axis in rodents [54]. Amphetamine exposure tests stimulated high rates of ACTH release using dosages as low as $6 \mu \mathrm{g} / \mathrm{kg}$ while cocaine induced HPA activation increased cortisol levels within 10 minutes of injection [54, 55]. Moreover cocaine use in rodents was found to release CRF from hypothalamic tissue in vitro; this demonstrates that psychostimulant drugs mediate their effects on the HPA through CRF to induce stress events in the brain. This subsequently caused abnormal increases of ACTH and cortisol in the CSF and blood stream [54].

Broader studies testing the impact of alcohol and opioids demonstrated similar results suggesting that many forms of substance abuse cause damage to the HPA system where prolonged exposure would induce long term HPA dysregulation [12]. Chronic administration of cocaine in subsequent studies was found to successfully cause HPA over-stimulation implicating the inability of the HPA axis to raise tolerance response barriers against prolonged cocaine abuse [56]. Protracted use would likely induce glucocorticoid burden leading to organ system damage as seen in chronic cocaine users [12].

The initial CRF response to alcohol was a 3 fold increase in CRF concentration in the CSF. However the development of a tolerance barrier was suspected as PVN CRF neurons showed a reduction in CRF secretion. This contradicted observations in the pituitary which demonstrated a 2 fold increase in CRF secretion. Conclusions from the study determined that long term alcohol exposure engendered atypical HPA hyperactivity.

Substance abusers in withdrawal commonly express the symptoms of negative and illogical behaviour such as dysphoria, depression and irritability. Clinical and non-clinical research has indicated that stress, depression and negative mood states are strong confounding factors that increase the likelihood of perpetuation of drug use in substance abusers $[12,57]$. Clinical trials provided circumstantial evidence by demonstrating that heroin experienced mice displayed "drugseeking" behaviour after being exposed to a stress event [55, 57]. Survey statistics established that many substance abusers were unable to break addictions due to psychological stress, depression and negative mood states which derived from HPA dysregulation. 
The data obtained from these investigations leads to a theory that substance abuse is potentially a means of self-medication to stave off depression or negative mood states. Ironically the same behaviour demonstrated by narcotic addicts increases the stress placed on the brain resulting in a never ending cycle of CFR secretion and HPA dysregulation [56].

\section{Dexamethasone tests and glucocorticoids:}

The condition of hypercorticolism, the hypersecretion of cortisol, is a common trait seen in patients suffering from depression, anxiety and substance abuse. This condition relates to the abnormal secretion of cortisol due to excessive CRF activation of the HPA axis. Psychiatric patients uniformly fail to show any lasting response to the dexamethasone suppression tests which should inhibit CRF release. This suggests that increased glucocorticoidal concentrations by the introduction of dexamethasone did not aid in the inhibition of CRF release as expected. Two hypotheses put forward suggested the potential inception of a yet to be identified resistance mechanism against standard negative feed-back or the manifestation of an overactive HPA axis resulting in a HPA drive which was able to easily surpass any cortisol inhibition message [36].

CRF challenge tests were performed by injecting CRF and urocortin, a CRF antagonist into psychiatric and control patients to analyse the ACTH production response. Results showed diseased patients had a blunted ACTH response compared to control groups, suggesting that this was caused by down regulation of the pituitary $\mathrm{CRH}_{1} \mathrm{Rs}$ in diseased patients as indicated in previous post mortem studies. Though control groups had higher rates of ACTH synthesis in the short term during the experiment, diseased patients, still had a higher ACTH secretion rate in the long term due to the constant hypersecretion of CRF.

Investigations into hypercortisolism and its role in HPA dysregulation revealed that extreme cortisol concentrations lead to the increased expression of CRF mRNA in the bed nucleus of the stria terminalis, amygdala and PVN [4, 23]. These results provide a possible explanation for the $400 \%$ increase in CRF secreting neurons observed in the post mortem studies described earlier in this chapter. These observations that implicate the very pathway meant to inhibit CRF production can be a contributing instigator of additional CRF secretion. Thus, this suggests the presence of a positive feedback mechanism promoting HPA dysregulation and hypersecretion of CRF is established in diseased patients.

\section{Novel CRF antagonists}


With the wealth of evidence that has amassed implicating the role of the HPA-CRF system in the pathogenesis of affective, anxiety and drug related psychiatric conditions, attention has turned to seek a new generation of pharmaceutical agents targeting CRF receptors. The structural entities that possess CRF antagonistic activity have been reviewed,[58,59,60] however some of the more common and important are summarised here.

Initial attempts at $\mathrm{CRF}$ antagonist development resulted in the discovery of the oxopyrazoline thiocyanates (I) Fig. (3) [61], which showed weak activity against CRF (3-70 mM/L). Further adaptations led to the development of five-membered ring systems focusing on the thiazole core (II, III) Fig. (3) framework which has resulted in improved activity (15 nM/L) [61]. Improvements were also achieved via the addition of quinoline and other bulky side groups dramatically enhancing activities.

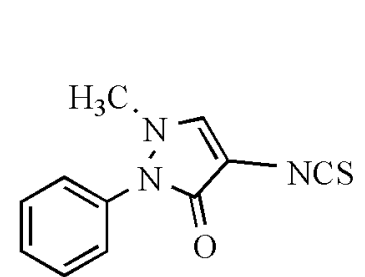

(1)<smiles>CCCN(c1nc(-c2ccc(Cl)cc2Cl)c(C)s1)C1CC1</smiles>

(II)<smiles>CCCN(c1nc(-c2ccc(Cl)cc2Cl)c(C)s1)c1cccc2ncccc12</smiles>

(III)

Figure 3: Examples of CRF antagonists containing a five-membered heterocyclic core unit.

CP-154,526 (IV) Fig (4) was the first potent non-peptide $\mathrm{CRF}_{1} \mathrm{R}$ specific antagonist to be developed [62]. This antagonist has demonstrated a binding preference for $\mathrm{CRF}_{1} \mathrm{R}$ having a binding activity of $2.7 \mathrm{nmol} / \mathrm{L}$ when tested in rat PVN neuronal tissue. Trials using this antagonist have shown it to be a potent inhibiter of CRF activation on the HPA axis reducing stress responses and also attenuating drug seeking behaviour in rodents [61] and human trials of this compound are imminent. 
<smiles>CCCCN(CC)c1nc(C)nc2c1c(C)cn2-c1c(C)cc(C)cc1C</smiles>

(IV)

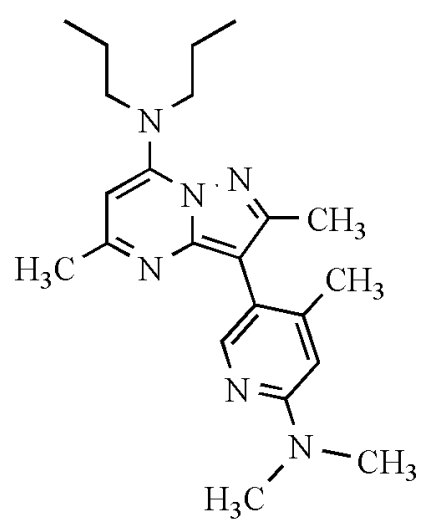

(V)

Figure 4: Selective $\mathrm{CRF}_{1} \mathrm{R}$ antagonists CP-154,526 (IV) and R121929 (V)

These alternative additional fused six-membered ring systems, namely aniline-pyrimidines structures such as R121919 (V) Fig (4) is a selective $\mathrm{CRF}_{1} \mathrm{R}$ antagonist which has shown remarkable benefits over previously synthesised compounds in its class due to increased solubility and its capacity to easily penetrate the blood brain barrier [63, 64]. Human clinical trials have shown that this compound improves anxiety and depression states. Clinical trials also provided evidence indicating its ability to attenuate HPA axis hyperactivity while not entirely inhibiting ACTH or cortisol release allowing for regular HPA related functions to continue unhampered [63, 64]. Beneficial outcomes have allowed R12919 (V) to move on to larger clinical trials however, these were discontinued due to hepatoxicity. [65] Research into the CRF and the HPA axis continues with initial steps giving more support to the HPA dysregulation hypothesis.

\section{Conclusion}

Compounding evidence from a wide range of pre-clinical and clinical studies has produced support for the HPA axis hypothesis suggesting hypersecretion of CRF has a profound effect in initiating psychiatric disabilities ranging from affective disorders to substance abuse. The source of increased CRF has been delegated to the hyperactivity of CRF releasing neurons within the amygdala, PVN and the pituitary causing increased rates of ACTH and cortisol secretion in rodents, primates and humans. In reply to these results drug developers have shown that experimental CRF antagonists might provide alternative therapeutic agents through regulation of the HPA axis.

\section{References}

[1] D. A. Regier, W. E. Narrow, D. S. Rae, R. W. Manderscheid, B. Z. Locke, F. K. Goodwin, Arch. Gen. Psychiat. 1993, 50, 85-94. 
[2] The Numbers Count: Mental Disorders in America

http://www.nimh.nih.gov/publicat/numbers.cfm (28/3/2006)

[3] Social Inequalities and the Distribution of the Common Mental Disorders. Ed. T. Fryers, R. Jenkins, D. Melzer, 2 Ed., Taylor \& Francis: New York, 2004.

[4] A. J. Mitchell, Neurosci. Biobehav. Rev. 1998, 22, 635-651.

[5] M. Pelleymounter, M. Joppa, M. Carmouche, M. J. Cullen, B. Brown, B. Murphy, D. E.

Grigoriadis, N. Ling, A. C. Foster, J. Pharm. Exp. Ther. 2000, 293, 799-806.

[6] T. W. Lovenberg, D. T. Chalmers, L. Changlu, E. B. De Souza, Endocrinol. 1995, 136, 41394142.

[7] R. L. Leahy, S. J. Holland, Treatment Plans and Interventions for Depression and Anxiety Disorders. 1st Ed, The Guilford Press: New York, 2000.

[8] D. K. Grammatopoulos, G. P. Chrousos, Trends Endocrin. Met. 2002, 13, 436-444.

[9] S. M. Stahl, Essential Psychopharmacology. 3rd Ed., Cambridge University Press: Cambridge, 1998.

[10] K. Lambert, C. H. Kinsley, Clinical Neuroscience. 1st Ed., Worth Publishers: New York, 2004 [11] M. Townsend, Essentials of Psychiatric Mental Health Nursing. 3rd Ed., F.A. Davis: Philadelphia, 2005

[12] Z. Sarnyai, Y. Shaham, S. C. Heinrichs, Pharmacol. Rev. 2001, 53 (2), 209-243.

[13] N. R. Carlon, Foundations of Physiological Psychology. 6th Ed., Pearson Education: Boston, 2005

[14] G. Baker, W. Dewhurst, Biochemical Theories of Affective Disorders. 2 Ed., Croom Helm: London, 1985

[15] C. Nmeroff, Sci. Am. 1998 278, 28-35.

[16] H. Rang, M. Dale, J. Ritter, P. K. Moore, Pharmacology. 5th Ed., Churchill Livingstone: Loanhead, 2003.

[17] A. Frazer, J. Clin. Psychopharm. 1997, 17, Suppl 1, 2S-18S.

[18] M. Wong, J. Licinio, Nat. Rev. Neurosci. 2001, 2, 343-351.

[19] A. Savinelli, Multidisciplinary Association for Psychedelic Studies Letters 1995, 6 (1), 58.

[20] R. Pies, J. Clin. Psychopharm. 1995, 15, 303-305.

[21] I. Maidment, Psychiatr. Bull. 2000, 24, 348-351.

[22] C. Salzman, J. Psychiat. Res. 1993, 27, Suppl 1, 97-110.

[23] B. DeSouza, D. Grigoriadis, Neuropsycopharmacology: The Fifth Generation progress. 5 Ed., 2002, p 19.

[24] M. P. Conn, H. M. Goodman, A. Cherrington, L. S. Jefferson, J. L. Kostyo, The Endocrine System. 1st Ed., Oxford University Press: New York, 2001 
[25] D. K. Grammatapoulos, E. W. Hillhouse, Lancet 1999, 354, 1546-1549.

[26] G. Aguilera, M. A. Millan, R. L. Hauger, Annal. NY Acad. Sci. 1987, 512, 48-66.

[27] E. Ur, A. Grossman, Acta Endocrinol. 1992, 127, 193-199.

[28] T. L. Bale, W. W. Vale, Ann. Rev. Pharmacol. 2004, 44, 525-557.

[29] P. J. Gilligan, D. W. Robertson, R. C. Zaczek, J. Med. Chem. 2000, 43, 1641-1660.

[30] E. B. De Souza, J. Neurosci. 1987, 7, 88-100.

[31] D. E. Grigoriadis, X. J. Liu, J. Vaughn, Mol. Pharmacol. 1996, 50, 679-686.

[32] E. B. De Souza, E. L. Webster, D. E. Grigoriadis, D. E. Tracey, Psychopharmacol. Bull. 1989, 25, 299-305.

[33] F. Holsboer, J. Psychiat. Res. 1998, 33, 181-214.

[34] D. N. Orth, Endocr. Rev. 1992, 13, 164-191.

[35] S. P. Malkoski, R. I. Dorin, Mol. Endocrinol. 1999, 13, 1629-1644.

[36] M. J. Perone, C. A. Murray, O. A. Brown, S. Gibson, A. White, E. A. Linton, A. V. Perkins, P. R. Lowenstein, M. G. Castro, Mol. Cell Endocrinol. 1998, 142, 191-202.

[37] G. Aguilera, Trends Endocrin. Met., 1998, 9, 329-336.

[38] E. J. Nestler, M. Barrot, R. J. Di Leone, A. J. Eisch, S. J. Gold, L. M. Monteggia, Neuron 2002, 34, 13-25.

[39] H. Lehnert, C. Schulz, K. Dieterch, Neurochem. Res. 1998, 23, 1039-1052.

[40] K. Itoi, A. F. Seasholtz, S. J. Watson, Endocrinol. J. 1998, 45, 13-33.

[41] M. J. Owens, C. B. Nemeroff, Pharmacol. Rev., 1991, 43, 425-473.

[42] C. B. Nemeroff, G. Bissette, A. C. Andorn, M. Stanley, Arch. Gen. Psychiatry. 1988, 45, 577579.

[43] F. C. Raadsheer, W. J. Hoogendijk, F. C. Stam, F. J. Tilders, D. F. Swaab, Neuroendocrinology 1994, 60, 436-444.

[44] F. Raadasheer, K. Heerikhuize, J. Lucassen, W. Hoogendijk, F. Tilders, D. Swaab, Am. J. Psychiat. 1995, 152, 1372-1376

[45] C. M. Banki, L. Karmacsi, G. Bissette, C. B. Nemeroff, J. Affect. Disorders 1992, 25, 39-45.

[46] C. B. Nemeroff, G. Bissette, H. Walleus, I. Karlsson, K. Eklund, C. D. Kilts, P. T. Loosen, W. W. Vale, Science 1984, 226, 1342-1344.

[47] L. Arborelius, M. J. Owens, P. M. Plotsky, C. B. Nemeroff, J. Endocrinol. 1999, 160, 1-12.

[48] F. Holsboer, A. Gerken, U. von Bardekeben, W. Grimm, H. Beyer, O. A. Muller, G. K. Stalla, Biol. Psychiatry. 1986, 21, 601-611.

[49] F. Holsboer, C. J. Lauer, W. Schreiber, J.-L. Krieg, Neuroendocrinology 1995, 62, 340-347.

[50] M. Joels, E. R. de Kloet, Prog. Neurobiol. 1994, 43, 1-36. 
[51] K. Inoue, G. R. Valdez, T. M. Reyes, L. E. Reinhardt, A. Tabarin, J. Rivier, W. W. Vale, P. E. Sawchenko, G. F. Koob, J. Pharmacol. Exp. Ther. 2003, 305, 385-393.

[52] M. A. Pelleymounter, M. Joppa, M. Carmouche, M. J. Cullen, B. Brown, B. Murphy, D. E. Grigoriadis, N. Ling, A. C. Foster, J. Pharmacol. Exp. Ther. 2000, 293, 799-806.

[53] T. Skutella, J. C. Probst, U. Renner, F. Holsboer, C. Behl, Neuroscience 1998, 85 (3), 795-805.

[54] S. Aston-Jones, G. Aston-Jones, G. F. Koob, Psychopharmacology 1984, 84, 28-31.

[55] H. A. Baldwin, S, Rassnick, J. Rivier, G. F. Koob, K. T. Britton, Psychopharmacology 1991, 103, 227-232.

[56] M. E. Carroll, R. A. Meisch, Adv. Behav. Pharmacol. 1984, 4, 47-88.

[57] P. A. Iredale, J. D. Alvaro, Y. Lee, R. Z. Terwillinger, Y. L. Chen, R. S. Dunman, J. Neurochem. 2000, 74, 199-208.

[58] A. McClusky, J. Garner, P. A. Keller, Bioorg. Med. Chem. 2000, 8, 1213-1223.

[59] M. Lanier, J. P. Williams, Expert Opin. Ther. Pat., 2002, 12, 1619-1630.

[60] C. Contoreggi, K. C. Rice, G. Chrousos, Neuroendocrinology, 2004, 80, 111-123.

[61] D. Grigoriadis, M. Haddach, N. Ling, J. Saunders, Curr. Med. Chem. - CNS Agents 2001, 1, 63-97.

[62] D. W. Shulz, R. S. Mansbach, J. Sprouse, J. P. Braselton, J. Collins, M. Corman, A. Dunaiskis, S. Faraci, A. W. Schmidt, T. Seeger, P. Seymour, F. D. Tingley, Proc. Natl. Acad. Sci. USA 1996, 93, 10477-10482.

[63] A. W. Zobel, T. Nickel, H. E. Kunzel, N. Ack1, A. Sonntag, M. Ising, F. Holsboer, J. Psychiatry Res. 2000, 34, 171-181.

[64] D. A. Gutman, M. J. Owens, K. H. Skelton, K. V. Thrivikraman, C. B. Nemeroff, J. Pharmacol. Exp. Ther. 2003, 304, 874-880.

[65] C. F. Gillespie, C. B. Nemeroff, Psychosom. Med. 2005, 67, Suppl 1: S26-S28. 